\title{
O Percurso e seus Percalços: Itinerário Terapêutico nos Transtornos Alimentares
}

\author{
Élide Dezoti Valdanha-Ornelas ${ }^{1}$ \\ Manoel Antônio dos Santos \\ Universidade de São Paulo
}

\begin{abstract}
RESUMO - Este estudo teve por objetivo delimitar o itinerário terapêutico - busca por cuidados à saúde, diagnóstico e tratamento - percorrido por mães cujos filhos foram diagnosticados com anorexia nervosa (AN). Trata-se de estudo do tipo exploratório, descritivo e transversal, no enfoque qualitativo de pesquisa. Para a coleta de dados, foram realizadas entrevistas semiestruturadas. A análise de conteúdo temática foi utilizada como estratégia de organização dos dados. Os resultados apontaram diversas barreiras no acesso a serviços especializados para o tratamento da AN. O limitado conhecimento dos profissionais acerca dessa psicopatologia retarda o diagnóstico, o que tem consequências negativas para o prognóstico. O conhecimento produzido sobre a trajetória de busca de ajuda para o problema pode contribuir para aprimorar a oferta e qualificação dos cuidados oferecidos.
\end{abstract}

Palavras-chave: transtornos alimentares, anorexia nervosa, itinerários terapêuticos, tratamento, processos terapêuticos

\section{Pathways and Pitfalls: A Therapeutic Itinerary in Eating Disorders}

\begin{abstract}
This study aimed to delimit the therapeutic itinerary - the search for healthcare, diagnosis and treatment - taken by mothers whose children were diagnosed with anorexia nervosa (AN). This is an exploratory, descriptive cross-sectional study with a qualitative approach. A semi-structured interview was used for data collection. Thematic content analysis was used to organize the data. The results indicate there are many barriers in accessing specialized services for the treatment of AN. The limited knowledge of professionals concerning this psychopathology delays diagnosis, which has negative consequences for prognosis. Knowledge acquired in this field will contribute to improve the delivery and qualification of the available care.
\end{abstract}

Keywords: eating disorders, anorexia nervosa, therapeutic itineraries, treatment, therapeutic processes

O Manual Diagnóstico e Estatístico de Transtornos Mentais (DSM-V) (Associação Americana de Psiquiatria, 2013) e a Classificação Internacional de Doenças (CID-10) (Organização Mundial de Saúde, 1993) apontam, como principais transtornos alimentares (TAs), a anorexia nervosa (AN) e a bulimia nervosa (BN). Esses quadros têm em comum comportamento alimentar gravemente perturbado, tentativa de controle excessivo do peso corporal por meios não apropriados e marcada distorção da imagem corporal. Especialmente na AN, o paciente vivencia um medo patológico de engordar, embora seu peso esteja abaixo do ideal (Bighetti et al., 2007; Cartwright, 2004; Claudino \& Borges, 2002; Leonidas \& Santos, 2013; Saikali, Soubhia, Scalfaro, \& Cordás, 2004).

O desenvolvimento da AN pode estar associado a alterações fisiológicas importantes, tais como distúrbios endócrinos (por exemplo, amenorreia em algumas mulheres), alterações gastrointestinais, cardiovasculares, hematológicas e metabólicas, que atuam também como mantenedoras da doença, podendo, em casos extremos, levar à morte (Dunker \& Philipi, 2003; Morgan, Vecchiatti, \& Negrão, 2002). Estudos consideram a AN como o transtorno psiquiátrico que apresenta maior taxa de mortalidade (Birmingham, Su, Hlynsky, Goldner, \& Gao, 2005; Morgan \& Claudino, 2005). Os TAs podem ser considerados condições crônicas de

1 Endereço para correspondência: LEPPS, Departamento de Psicologia, Faculdade de Filosofia, Ciências e Letras de Ribeirão Preto, Avenida Bandeirantes, 3900, Monte Alegre, Ribeirão Preto, SP, Brasil. CEP 14040-901.E-mail: elidevaldanha@usp.br saúde, devido ao seu longo curso, e apresentam altos índices de comorbidade psiquiátrica, sobretudo com transtornos bipolares, depressivos, de personalidade e de ansiedade (APA, 2013; Leonidas \& Santos, 2014; Rosa \& Santos, 2011).

Nos Estados Unidos a prevalência da AN varia em torno de $2 \%$ a $5 \%$ em mulheres adolescentes e adultas, sendo a terceira doença mais comum entre adolescentes, perdendo apenas para a obesidade e a asma (Dunker \& Phillipi, 2003). Já para Morgan e Claudino (2005), a prevalência de AN entre mulheres jovens tem taxas médias de, aproximadamente, $0,3 \%$, e a de $\mathrm{BN}, 1 \%$. Ao revisarem estudos de diversas nacionalidades sobre a prevalência dos TAs, Santana, Ribeiro Junior, Giral e Raich (2012) verificaram que há variações epidemiológicas de $0,3 \%$ a $2,2 \%$ para $\mathrm{AN}$ e de $0,1 \%$ a $2 \%$ para a BN.

De acordo com a American Psychiatry Association (2013), estudos apontam que TAs são mais comuns em pessoas do sexo feminino, estimando-se a prevalência homem-mulher com variações entre 1:6 e 1:10 (Andrade \& Santos, 2009; APA, 2006). Segundo a American Dietetic Association Reports - ADA (2011), a maior incidência de casos de $\mathrm{AN}$ e $\mathrm{BN}$ acontece na adolescência.

A escassez de estudos nacionais que apresentem dados de prevalência de TAs pode ser explicada pela baixa fidedignidade de registros de pacientes diagnosticados na rede pública de saúde. Além disso, uma minoria de pessoas busca tratamento especializado para esses transtornos mentais. No Brasil, não existem estudos populacionais que informem a prevalência desses quadros, o que dificulta o 
planejamento de estratégias interventivas e programas de prevenção e promoção de saúde. Um fator que contribui para manter essa lacuna no contexto nacional é o fato de que são necessários estudos de longa duração para a obtenção de dados epidemiológicos (Alves, Vasconcelos, Calvo, \& Neves, 2008; Nunes, 2006).

$\mathrm{Na}$ era contemporânea, a visibilidade social conferida à $\mathrm{AN}$ e à $\mathrm{BN}$, bem como os significativos graus de morbidade e mortalidade que acompanham essas psicopatologias, têm convertido gradualmente esses quadros em importante problema de saúde pública. Essa constatação instaura um desafio, pois exige que o profissional domine um conhecimento teórico e técnico específico que o habilite a lidar com os desafios que essa clínica impõe (APA, 2006; Andrade \& Santos, 2009; Bighetti et al., 2007; Rosa \& Santos, 2011).

São considerados fatores desencadeadores e mantenedores do quadro psicopatológico: o meio sociocultural (por exemplo, influência das mensagens disseminadas pela mídia ou pelo círculo social), a dinâmica familiar (indiferenciação psíquica entre os membros do grupo, perda das fronteiras individuais, confusão de papéis e funções) e aspectos da personalidade do indivíduo (sentimento generalizado de insatisfação, afetos negativos), o que caracteriza a AN como um transtorno mental de etiologia multifatorial (Appolinário \& Claudino, 2000; Bighetti et al., 2007; Chan \& Ma, 2004; Nilsson, Abrahamsson, Torbiornsson, \& Hägglöff, 2009; Oliveira \& Santos, 2006; Polivy \& Herman, 2002; Santana et al., 2012).

Após o início dos sintomas de AN, a pessoa acometida pode perder alguns de seus papéis funcionais, no que concerne ao trabalho, às amizades e às atividades de lazer (Quiles-Cestari \& Ribeiro, 2012). Esses pacientes tendem a negar a gravidade de sua psicopatologia ou mesmo sua existência, especialmente na etapa inicial do transtorno. Seus principais aspectos de personalidade estão relacionados à baixa autoestima, introversão, insegurança, inadequação, perfeccionismo e obsessividade. Tendem a demonstrar dificuldades no estabelecimento de vínculos afetivos, acentuada inibição e retraimento social, o que torna seu círculo social restrito e empobrecido (Leonidas \& Santos, 2012; Leonidas, Crepaldi, \& Santos, 2013; Morgan et al., 2002).

$\mathrm{O}$ ambiente familiar de pessoas com AN é permeado por tentativas recorrentes de controle de comportamentos, com estilos de comunicação negativos e expressões de linguagem de mesma natureza, associados à discrepância nos valores e nas normas familiares, evidenciando as confusões vivenciadas no cotidiano (Benninghoven, Tetsch, Kunzendorf, \& Jantscheck, 2007; Cobelo, Saikali, \& Schomer, 2004; Dallos \& Denford, 2008; Latzer, Ben-Ari, \& Galimidi, 2002). Quando um membro da família desenvolve TAs, os demais se reorganizam em torno de suas necessidades e vulnerabilidades. Assim, emerge uma nova configuração familiar, em que o casal parental devota mais atenção ao filho acometido. Os outros filhos tendem a receber menos cuidados, o que pode fazer aflorar outras situações de conflito no lar. Os irmãos podem vivenciar sentimentos de abandono e desamparo, sentindo-se excluídos do grupo familiar. Nesse cenário adverso, a pessoa com TAs é, muitas vezes, percebida e descrita como um "novo"membro do grupo familiar, visto que passa a ocupar um espaço significativo nesse contexto, devido à atenção que demanda de toda a família (Areemit, Katzman, Pinhas, \& Kaufman, 2010; Honey,Clarke, Halse, Kohn, \& Madden, 2006; Latzer et al., 2002; Valdanha, Scorsolini-Comin, \& Santos, 2013a).

Devido à instalação geralmente insidiosa do problema, os membros do núcleo familiar podem demorar a perceber o desenvolvimento dos sintomas. A pessoa acometida sente vergonha dos sintomas ou não os considera relevantes e, assim, tenta camuflá-los perante os outros (Fursland \& Watson, 2014). Dessa forma, percebe-se uma significativa lacuna no período decorrido desde o início dos sintomas até o estabelecimento do diagnóstico e a instituição do plano terapêutico, nas suas múltiplas facetas: médica, nutricional, psicológica. A demora na busca por ajuda contribui para agravar o quadro clínico do paciente (Assumpção \& Cabral, 2002; Pinzon \& Nogueira, 2004). Estudo de Palma, Dos Santos e Ribeiro (2013), que avaliou pacientes que já necessitaram de hospitalização para tratamento de TAs, encontrou variações entre um e 240 meses desde o início dos sintomas até o estabelecimento do diagnóstico. Devese acrescentar, ainda, a escassez de serviços especializados e a carência de profissionais devidamente treinados e qualificados. Esse desconhecimento prejudica uma avaliação pertinente dos sintomas alimentares (Fursland \& Watson, 2014). Não temos conhecimento de estudos nacionais que apresentem um mapeamento de locais de tratamento especializado em TAs na rede pública de saúde. Um levantamento informal realizado na Internet aponta que existem cerca de oito programas e serviços multiprofissionais disponíveis com essas características - especializados e gratuitos. $\mathrm{O}$ grande número de pacientes que aguardam em filas de espera sugere que a oferta de serviços não supre a demanda.

Os pais dos jovens acometidos pela $\mathrm{AN}$, ao primeiro contato com o diagnóstico, tendem a se mostrar incrédulos, perplexos e assustados (Souza, Santos, \& ScorsoliniComin, 2009). Sentimentos de impotência, raiva, tristeza, medo e culpa permeiam o cotidiano dessas famílias após o acometimento, evidenciando o sofrimento familiar (Souza \& Santos, 2009). O sistema familiar precisa se readaptar à nova realidade que se instaura, de modo a proporcionar os cuidados necessários ao paciente. O contato com serviços ambulatoriais ou internações resulta em mudanças na rotina doméstica, podendo suscitar desorganização e exacerbação da angústia. Por outro lado, a continuidade do tratamento e da assimilação da rotina assistencial proporciona maior conhecimento do familiar acerca do transtorno, atenuando a ansiedade inicial frente ao desconhecido, o que contribui para o oferecimento de melhores condições de cuidado (Espíndola \& Blay, 2009; Souza \& Santos, 2010; Valdanha et al., 2013a). Esses achados sugerem a importância de ampliar o conhecimento produzido sobre a busca de ajuda profissional no contexto dos TAs.

O termo itinerário terapêutico pode ser compreendido como a busca por cuidados terapêuticos (diagnóstico e tratamento). Os estudos que utilizam esse constructo têm como propósito descrever quais são os caminhos percorridos por pacientes e familiares (usuários dos serviços de saúde) 
na tentativa de sanarem seus problemas de saúde (Gerhardt, 2006). Trata-se da construção gradual de um processo de escolha, avaliação e adesão a determinado tratamento de saúde.

Recente estudo de revisão da literatura sugere que as pesquisas com foco no itinerário terapêutico de usuários de serviços públicos de saúde no Brasil são recentes. As investigações, em geral, estão relacionadas à busca por tratamento para doenças crônicas ou psiquiátricas que requerem presença de acompanhante, o que enfatiza a necessidade de que todo o grupo familiar seja assistido em suas necessidades em relação aos cuidados com o paciente (Cabral, Martinez-Hemáez, Andrade, \& Cherchiglia, 2011).

$\mathrm{O}$ interesse em realizar o presente estudo, focalizando o itinerário terapêutico no contexto dos TAs, surgiu da observação e escuta rotineira de queixas reiteradas de pacientes e familiares, ingressantes em um serviço multidisciplinar especializado no atendimento a pessoas com TAs, em relação à demora para encontrarem tratamento especializado e eficaz e às barreiras enfrentadas. Essas barreiras, muitas vezes, culminam no diagnóstico errôneo ou tardio, em prejuízo do prognóstico e da evolução favorável do quadro clínico.

O conhecimento sobre os itinerários terapêuticos de usuários do serviço público de saúde pode contribuir para o entendimento de como as pessoas buscam e encontram tratamento e de como utilizam esses recursos, aproximandoas de um cuidado adequado e mais condizente com suas reais necessidades (Favero-Nunes \& Santos, 2010; Guerin, Rossoni, \& Bueno, 2012; Marques \& Mângia, 2013). Assim, entende-se que conhecer os caminhos que as pessoas diagnosticadas com AN e seus cuidadores/familiares percorrem tipicamente na busca por tratamento pode fornecer subsídios valiosos para a qualificação da assistência.

Percebe-se ainda a difusão de muitas concepções equivocadas acerca dos TAs junto à população - muitas vezes, de cunho moralista -, o que pode prejudicar a procura e o acesso a serviços especializados nessa psicopatologia (Leal, 2013; Gazignato, Scorsolini-Comin, Souza, Kazan, \& Santos, 2008; Souza \& Santos, 2014; Souza et al., 2009). Na fase inicial dos sintomas, os familiares geralmente atribuem as possíveis "causas" dos TAs às pressões da mídia pela magreza. Com o decorrer do tratamento, passam a refletir sobre aspectos externos (meio sociocultural) e internos (conflitos emocionais) que podem estar relacionados ao desenvolvimento da psicopatologia. Por vezes, os pais experimentam sentimentos de culpa em relação à recusa alimentar dos filhos (Souza et al.,2009), buscando identificar algum comportamento ou valores pessoais que supostamente teriam contribuído para o aparecimento do problema. Todos esses fatores mencionados podem estar relacionados ao surgimento dos TAs, no entanto, o modo como se dá a apropriação, pelos familiares, dos possíveis desencadeadores pode variar ao longo do tratamento. Assim, compreender a natureza multifatorial do problema é um desafio também para as famílias (Souza \& Santos, 2007).

Compreender que fatores podem facilitar ou dificultar o acesso de pacientes e familiares ao serviço de atendimento para os TAs pode contribuir para inserir melhorias no plano da divulgação do serviço junto à população, bem como no acesso da comunidade aos recursos disponíveis, reduzindo o risco de diagnósticos imprecisos, internações desnecessárias e incremento do sofrimento familiar em decorrência de "não se saber" com clareza o que está acontecendo com o membro acometido. Por outro lado, a disseminação de informações sobre os TAs junto à população merece uma reflexão crítica, já que não se encontra consenso entre os pesquisadores da área. A divulgação dos sintomas dos TAs na mídia, por exemplo, nem sempre tem um efeito esclarecedor sobre a população, mas pode, pelo contrário, fomentar temores, desconfianças e generalizações indevidas, resultando em tentativas de controle sobre a saúde em uma perspectiva de gerenciamento de riscos (Spink, Medrado, \& Mello, 2002).

Ao considerar esses pressupostos, este estudo teve por objetivo delimitar o itinerário terapêutico percorrido por mães cujos(as) filhos(as) foram diagnosticadas com AN e compreender as facilidades e barreiras encontradas no acesso aos serviços de saúde.

\section{Método}

Trata-se de um estudo do tipo exploratório, descritivo e transversal, desenvolvido sob o enfoque qualitativo de pesquisa. Esse delineamento de pesquisa vem ganhando espaço no campo científico da saúde, inclusive entre profissionais da área médica, com o intuito de se obter melhor compreensão da história de vida dos usuários dos serviços de saúde (Turato, 2005).

O desenho utilizado é de estudos de casos múltiplos. Cada caso é representado por uma mãe, que narra a trajetória que percorreu junto aos equipamentos de saúde, em busca de recursos de ajuda para sua/seu filha(o), até a chegada no serviço especializado de tratamento para TAs. Tal estratégia metodológica se mostra interessante para alcançar os objetivos propostos porque permite a apreciação de características comuns e incomuns entre os casos (Stake, 2000).

$\mathrm{Na}$ condução do estudo, foram respeitados os preceitos éticos da pesquisa envolvendo seres humanos, com aprovação do projeto pelo Comitê de Ética em Pesquisa da instituição hospitalar. Para preservar o anonimato das participantes, nomes próprios foram substituídos por nomes fictícios e informações que poderiam comprometer o anonimato foram suprimidas ou mascaradas.

\section{Participantes}

Participaram do presente estudo seis mães de jovens diagnosticados(as) com AN, que se encontravam em tratamento ambulatorial em um serviço multiprofissional especializado com mais de três décadas de atendimento ininterrupto. O número de participantes foi definido de acordo com o critério de saturação dos dados, que preconiza que as entrevistas devam ser finalizadas no momento em que os dados empíricos se repetirem, não adicionando informações novas que possibilitem a compreensão do fenômeno investigado. 
O estudo foi desenvolvido no Grupo de Assistência em Transtornos Alimentares (GRATA) do Hospital das Clínicas da Faculdade de Medicina de Ribeirão Preto da Universidade de São Paulo. Criado em 1982, é reconhecido no cenário nacional como o primeiro grupo multidisciplinar de atendimento aos TAs. Desde então, o serviço tem oferecido ininterruptamente assistência gratuita à população. Fazem parte dessa equipe médicos nutrólogos, nutricionistas, psicólogos, psiquiatras, enfermeiros e estagiários do curso de graduação em Psicologia e Nutrição (Dos Santos, 2006). As entrevistas foram realizadas nas dependências do ambulatório, em sala reservada, em dia de retorno dos pacientes e suas cuidadoras/acompanhantes.

Para a escolha dos participantes, foram estabelecidos os seguintes critérios de inclusão: diagnóstico de AN estabelecido pela equipe multiprofissional, estar em tratamento no GRATA, ter acompanhante disponível para participar da pesquisa, com conhecimento sobre os caminhos percorridos desde o início dos sintomas até a chegada ao tratamento especializado. Todas as pessoas convidadas para participar do estudo eram mães, o que representa a realidade do serviço em questão, no qual as mães são as cuidadoras principais. A entrevista foi realizada apenas com as cuidadoras.

\section{Procedimentos de Coleta e Análise dos Dados}

Para alcançar os objetivos propostos, foi aplicado um roteiro de entrevista semiestruturada para investigar o caminho percorrido pelas famílias até encontrarem o serviço especializado. Os dados foram coletados em uma sala reservada do serviço especializado. Para assegurar maior fidedignidade dos dados colhidos e preservar a objetividade do estudo, as entrevistas foram audiogravadas mediante anuência das participantes.

A análise de conteúdo na modalidade temática (Bogdan \& Biklen, 1994) foi utilizada como estratégia de análise dos dados. Os resultados foram organizados em categorias, levando-se em conta a regularidade das respostas e os padrões convergentes e divergentes de conteúdo dos relatos.

\section{Resultados e Discussão}

Das seis mães entrevistadas, cinco tinham filhas adolescentes ou jovens adultas. Apenas uma era mãe de adolescente do sexo masculino. As seis mães eram as acompanhantes principais de seus filhos nos dias de retorno ambulatorial desde o início do tratamento. A Tabela 1 apresenta os dados sociodemográficos das participantes, bem como a idade dos filhos e o tempo de tratamento no serviço especializado. As participantes tinham idades entre 42 e 52 anos, nível de escolaridade médio, eram, em sua maioria, casadas, exerciam atividade remunerada (destacandose a profissão de cozinheira), declararam-se católicas e frequentavam o serviço por um período que variava de dois meses a cinco anos.

Emergiram as seguintes categorias temáticas: Percepção dos sintomas iniciais da $\mathrm{AN}$ (orgânicos e psíquicos), Querendo entender o que acontece (crenças e significados atribuídos à sintomatologia), A procura por ajuda efetiva (que culmina com a experiência do diagnóstico), Falta de apoio dos familiares, Mudando a percepção sobre os TAs.

\section{Percepção dos Sintomas Iniciais da AN: "Eu achava que aquilo era normal"}

Essa categoria abrange o período pré-diagnóstico. Abarca as percepções das mães e de outros familiares em relação ao membro acometido pelo transtorno.

Ressalta-se que os sintomas de AN emergem primordialmente na adolescência, período de transição psicossocial que se caracteriza por inúmeras mudanças corporais, psicológicas e familiares (APA, 2013; Andrade \& Santos, 2009; Lima, Rosa, \& Rosa, 2012; Rosa \& Santos, 2011). Essas mudanças se refletem diretamente no

Tabela 1. Características sociodemográficas das participantes, idade dos filhos e tempo de tratamento.

\begin{tabular}{|c|c|c|c|c|c|c|c|}
\hline Mães & Idade & Estado civil & Escolaridade & Profissão & Religião & $\begin{array}{l}\text { Idade da(o) } \\
\text { filha(o) }\end{array}$ & $\begin{array}{c}\text { Tempo de } \\
\text { tratamento } \\
\text { no serviço }\end{array}$ \\
\hline Amanda & 46 & Casada & E. F. completo & Cozinheira & Evangélica & 26 & 2 meses \\
\hline Betina & 45 & Casada & $\begin{array}{c}\text { E. M. } \\
\text { completo }\end{array}$ & Cozinheira & Católica & 18 & 5 anos \\
\hline Célia & 51 & Casada & $\begin{array}{c}\text { E. M. } \\
\text { completo }\end{array}$ & Cozinheira & Católica & 25 & 2 anos \\
\hline Débora & 52 & Divorciada & E. F. completo & $\begin{array}{l}\text { Cozinheira } \\
\text { (aposentada) }\end{array}$ & Evangélica & 29 & 1 ano \\
\hline Eliana & 42 & Viúva & E. S. completo & Comerciante & Católica & 15 & 10 meses \\
\hline Fabiana & 45 & Separada & E. S. completo & Educadora & Católica & 13 & 8 meses \\
\hline
\end{tabular}

Nota. E. F.: Ensino Fundamental; E. M.: Ensino Médio; E. S.: Ensino Superior 
funcionamento familiar, que precisa se reorganizar para enfrentar as demandas que emergem nessa nova etapa do ciclo vital.

Uma das mães (Fabiana) relata ter percebido prontamente a restrição alimentar da filha. A adolescente tinha apenas 12 anos quando iniciaram os primeiros comportamentos alimentares restritivos. Nessa época, a mãe estava muito atenta à alimentação da jovem devido à sua idade, por entender que ela ainda necessitava desse tipo de cuidado. Nas famílias das outras mães entrevistadas, porém, os sinais e sintomas de AN só foram percebidos tardiamente, quando já estavam plenamente consolidados e circunscritos no corpo, que denunciava, por meio da extrema magreza, que algo de "anormal" estava acontecendo.

A filha de Betina e o filho de Eliana chegaram ao serviço com Índice de Massa Corporal (IMC) abaixo de 14, ou seja, em um estado de desnutrição grau 3 , situação considerada de elevado risco à saúde (a faixa de IMC considerada normal varia de 18 a $25 \mathrm{~kg} / \mathrm{m}^{2}$ ). A instalação dos problemas físicos e psíquicos, em casos como esses, é percebida como gradual, insidiosa e impalpável. Frequentemente, é um indivíduo do ambiente familiar que primeiro percebe os sinais de que há algo que não está indo bem:

Todo mundo achava ela magrinha demais e falava: "ai, essa menina deve ter anorexia", e eu falava: "ela não tem nada, ela come, ela só não come carne", mas a hora que eu comecei a prestar atenção no que a [nome da filha] comia, nossa, ela já estava... (Amanda)

Em alguns casos, a percepção se dá por um familiar, não necessariamente a mãe e, em nenhum caso, o pai: "Foi o irmão dela que falou: 'mãe, ah, mãe, leva ela no médico, que tem alguma coisa errada, o maiô dela tá caindo, ela emagreceu demais" (Betina).

O desconhecimento dos sinais e sintomas de Tas é um fator que dificulta a percepção da perturbação do comportamento alimentar nos estágios iniciais do transtorno:

No começo, eu não achei que o [nome do filho] estava com anorexia, porque, pra mim, anorexia era aquela doença que a pessoa comia uma folha de alface e vomitava ela, né. Achava que a anorexia já tinha a ver com a bulimia. E aí, quando o [nome do filho] teve anorexia, eu não acreditava porque ele comia muitas alfaces, muitas, um pratão de alface, e fazia muito esporte. (Eliana)

As vinhetas apresentadas apontam que as mães subestimaram a perda inicial de peso de seus filhos ou estavam pouco atentas às alterações de seus hábitos alimentares. Aos poucos, as mães foram percebendo algumas excentricidades nos hábitos alimentares, como seletividade e monotipia na escolha dos alimentos. Os jovens acometidos passaram a evitar os ambientes comuns de alimentação, como compartilhar a mesa com a família durante as refeições, e assumiram metas radicais para a redução de peso, na busca obsessiva por um corpo magro e perfeito.

A pessoa acometida por AN começa a se isolar cada vez mais e a apresentar uma crescente perda de interesses por coisas que antes atraíam sua atenção. Além disso, mantém uma relação de amor e ódio com a alimentação. Quando os sintomas físicos se agravaram (ossos proeminentes, fraqueza física, desmaios frequentes, amenorreia para as mulheres), começaram as especulações a respeito do que poderia estar acontecendo. A AN não aparecia como uma opção entre as possíveis doenças aventadas inicialmente pelas famílias.

\section{Querendo Entender o que Acontece: "No início era uma coisa muito distante"}

Essa categoria temática diz respeito às especulações feitas pelos membros do grupo familiar ao se darem conta de que algo não ia bem com a(o) jovem acometida(o). Nessa categoria, estão englobadas também as crenças e significados atribuídos à sintomatologia no momento anterior ao estabelecimento do diagnóstico dos TAs.

Bom, pra falar a verdade só vi na televisão, não sabia o que era, só vi... Pela Internet, televisão, mas nunca imaginava que eu ia... Passava as reportagens das meninas que morriam de anorexia... Eu nunca... Nunca ia achar que ia acontecer com a minha filha, e foi muito dificil, sabe, ainda tá sendo dificil. (Débora)

Eu já tinha ouvido falar, mas nunca dei importância. Já tinha ouvido falar de modelo que morreu de anorexia, da princesa Diana, que tinha bulimia, né, mas nunca me interessei. (Amanda)

De vez em quando, você ouvia uma notícia, passava alguma foto, né, alguma reportagem na televisão de "tal modelo anoréxica”, mas tudo pra mim era um negócio distante, eu não sabia nada, nada... (Betina)

Os excertos de fala selecionados evidenciam que o pouco conhecimento que as mães tinham sobre os TAs antes do diagnóstico de seus filhos estava relacionado às notícias impactantes veiculadas pela televisão, como a morte de algumas modelos por inanição. Todavia, a AN sempre foi considerada um problema muito distante da família, uma doença de celebridades, que não afeta pessoas comuns. Alguns relatos evidenciam a incompreensão dos motivos que levariam uma pessoa a deixar de se alimentar ou provocar deliberadamente vômitos, mas esse era o máximo de reflexão a que essas mães se permitiam acerca dessa temática (Souza et al., 2009).

\section{A Procura por Ajuda Efetiva: “Os médicos não têm preparo pra isso"}

De acordo com Favero-Nunes e Santos (2010), a falta de compreensão e de informações a respeito do quadro clínico gera aflições, mas também esperança entre os familiares, especialmente os pais. Na percepção das entrevistadas, muitos profissionais despreparados minimizam os sintomas, atribuindo-os à "frescura", buscando assim atenuar a preocupação da família. Porém, na medida em que o quadro clínico se agrava, as angústias se avolumam e aumenta a necessidade dos pais de obter respostas adequadas às suas inquietações crescentes.

Uma das famílias (Amanda) descobriu que a jovem estava com AN quando ela foi internada às pressas em decorrência de um desmaio por fraqueza física. Inicialmente, Amanda pensou que sua filha pudesse ter anemia, mas afirma que não se preocupou a ponto de procurar um médico, uma vez que considera a anemia um quadro que não oferece risco 
e pode ser facilmente superado com alimentação correta, enriquecida com ferro. Outra mãe, Fabiana, contou que "já sabia" que a filha estava com AN ao observar a obsessão que a filha desenvolveu em relação à alimentação. Procurou o auxílio de um psiquiatra, amigo da família, que confirmou o diagnóstico inicial.

As outras quatro mães participantes relataram que fizeram uma verdadeira "peregrinação" por profissionais, sem encontrarem médicos habilitados a diagnosticarem o verdadeiro problema de suas filhas. Passaram por momentos de aflição e angústia, por perceberem que algo de errado estava acontecendo com as adolescentes, mas obtiveram do sistema de saúde poucas respostas convincentes e conclusivas frente aos seus questionamentos. Nessa vertente, nota-se que faltam locais não só para o acolhimento qualificado dos anseios dos cuidadores dos pacientes com AN, como também espaços de assimilação e apropriação dos saberes relacionados aos TAs, manejo e gestão dos sintomas, aquisição de estratégias de enfrentamento, face aos desafios impostos pela complexidade e natureza multifatorial do problema.

A percepção de que algo não vai bem com a(o) filha(o) leva as mães a empreenderem sucessivas buscas por serviços e profissionais, à procura de uma resposta para suas inquietações:

Era um desespero tão grande, porque eu não sabia o que era. Ai, pra você ter uma ideia, a gente levou ela no médico, pagou o médico do estômago, achava que era gástrico, fez a endoscopia, não dava nada. Fez tudo quanto era exame, não dava nada. E era o quê? Era transtorno alimentar, mas a gente não sabia. (Débora)

Ai passou por nutricionista, psicólogo, e falavam que era depressão, era depressão, era depressão [...]. Ai, um dia lá, fui num dentista e ela foi comigo [...], ai ele falou: "essa menina não tem depressão não, essa menina tem aquela doença lá, que a pessoa não come, uma tal de anorexia, procura um psiquiatra". Eu não voltei mais no dentista, porque me mandou procurar psiquiatra. (Célia)

Porque fui no médico em [menciona três cidades diferentes], eles não têm preparo pra isso. Eu acho que é muito novo até pra eles mesmo, né? Ai eles falavam: "É frescura! Sabe aquela época da adolescência? Deixa ela lá, morrer de fome não vai não, a hora que tiver fome ela pede". Eu falava: "não, não é frescura, a [nome da filha] não é frescurenta". (Betina)

As mães relataram ter experimentado frustração e desapontamento com os profissionais inicialmente consultados. Sentiam-se pouco compreendidas e, muitas vezes, negligenciadas nos cuidados recebidos, já que as resoluções oferecidas à(o) filha(o) doente eram ineficazes, corroborando a percepção das mães participantes do estudo de Keitel, Parisi, Whitney e Stack (2010). Os comportamentos de restrição alimentar e vômitos autoinduzidos eram tratados como quadros orgânicos, o que também dificultava o diagnóstico preciso do transtorno mental. Nessa direção, algumas mães esboçaram que não contaram com o apoio dos demais familiares na busca por tratamento, já que eles julgavam que esse cuidado não era necessário ou que não fazia sentido, o que contribuía para aumentar suas incertezas e incrementar ainda mais a ansiedade familiar.
Para os familiares, o momento do diagnóstico (e o período anterior ao seu estabelecimento) é vivenciado como um período de crise. A família é essencial na tomada de decisão a respeito do tratamento, especialmente quando se trata de adolescentes (Mattosinho \& Silva, 2007). No serviço especializado em apreço neste estudo, a participação de um familiar é obrigatória nos dias de retorno dos pacientes, o que modifica diretamente a rotina do núcleo familiar. Algumas funções familiares precisam ser redistribuídas e determinadas tarefas são remanejadas pelas mães, para que possam acompanhar as(os) filhas(os) no tratamento. A Figura 1 evidencia o caminho percorrido por pacientes e familiares desde o início dos sintomas até o princípio do tratamento no serviço especializado em TAs.

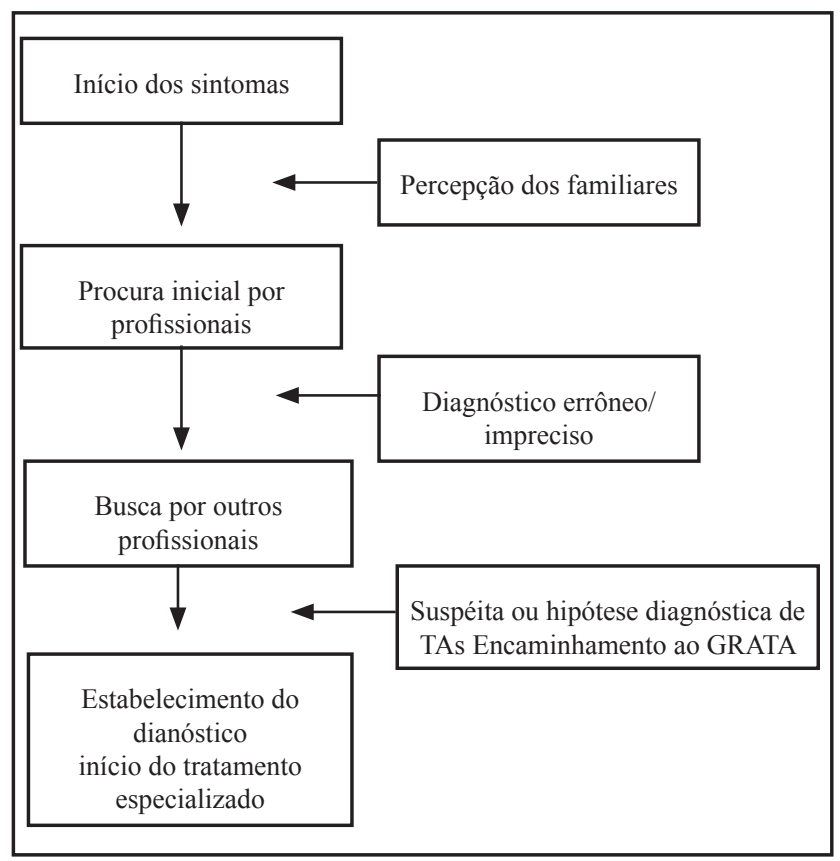

Figura 1. Caminho percorrido por pacientes e familiares até o início do tratamento em serviço especializado

\section{Falta de Apoio dos Familiares: "Ele não quer entender"}

Esta categoria abrange a percepção dos outros familiares em relação aos sintomas de TAs da(o) jovem acometida(o) e do tratamento realizado. Predominam reações emocionais de incredulidade frente aos sintomas, que levam a não aceitação do problema, em parte porque ele é identificado, no modelo biomédico, como uma "entidade psiquiátrica". Desse modo, recaem sobre os TAs os mesmos preconceitos e estigmas direcionados à doença mental.

Até que teve uma fase mais dificil, que é quando a [nome da filha] ficou internada. Ela ficou doente e ele [o pai] não aceitava a doença dela de jeito nenhum. A família também não aceitou. Então, só pra gente, assim, só pra aceitar o tratamento dela com psiquiatra foi um ano... que ela se agravou, ele falava: "porque ela não é louca, psiquiatra é pra louco" [...]. Eles [marido e outros filhos] não aceitavam muito a doença dela, então eles achavam muito que era sem-vergonhice, e que eu 
abandonei eles porque eu tava lá [acompanhando a filha na internação]. (Célia)

A gente conversa bastante, mas ele [pai] não quer entender. A [psicóloga da equipe] já ligou pra ele, pra convidar pra ele vir, pra participar, pra entender um pouco, mas ele acha que é uma frescura, que ela não tem nada [...] ele fala pra mim que ele não sabe o quê que eu venho fazer aqui. (Débora)

A não compreensão por parte dos pais e de outros familiares acerca da natureza psicopatológica dos sintomas frequentemente os leva a confundirem os TAs com "fraqueza de caráter", "frescura" ou tentativa deliberada de chamar a atenção para si. Essas crenças errôneas levam à procrastinação da busca por tratamento, à não aceitação da doença e, consequentemente, a um pior prognóstico.

Então, acho que a dificuldade é, assim, deles [familiares] não aceitarem que é difícil, não é um processo fácil, que não é curto, né, que é uma coisa longa. Eles [familiares] acham que é uma doença que vai sarar, tomou remedinho e sara, né, então acho que a maior dificuldade é o esclarecimento, que tá vindo agora, que é bem recente. (Fabiana)

A família é considerada como peça essencial no tratamento dos TAs. O núcleo familiar pode contribuir ao se empenhar para que a pessoa acometida não interrompa o tratamento, o que é muito comum no contexto dos TAs (Souza \& Santos, 2010). No presente estudo, foi possível perceber que, com o passar do tempo, a família passa a se sentir parte integrante do tratamento. Famílias ainda iniciantes nesse processo de reabilitação mostram dificuldades em compreender a psicopatologia, bem como em voluntariarse para auxiliar e acompanhar a(o) paciente no serviço. É um fato bem conhecido dos profissionais dos serviços que pacientes com TAs não procuram atendimento por conta própria, mas são levados por suas famílias, muitas vezes contra sua vontade (Scorsolini-Comin, Souza, \& Santos, 2010). A negação do problema por parte da(o) filha(o) encontra seu complemento no ceticismo dos pais. A equipe multiprofissional deve estar preparada para receber pacientes e familiares nessas condições e facilitar o acesso dos mesmos aos serviços prestados, devendo forjar um canal de comunicação eficaz com a família e a comunidade por intermédio do fortalecimento de um vínculo de confiança e respeito ao sofrimento do outro (Guerin et al., 2012; Valdanha, Scorsolini-Comin, Peres, \& Santos, 2013b).

A exemplo do que se observa nos serviços de TAs, as mães são as principais acompanhantes de filhas(os) com AN no tratamento (Marco \& Cantero, 2009). Atualmente se discute se a ausência dos homens nos serviços públicos de saúde está realmente vinculada a uma necessidade concreta de se ausentar do trabalho (por questões profissionais, por exemplo, ou até mesmo pelo deslocamento geográfico implicado) ou se está relacionado à falta de percepção de sua importância no processo saúde/doença/cuidado dos filhos (Gomes et al., 2011).

Dentre as participantes deste estudo, uma (Betina) era acompanhada pelo marido, enquanto as demais compareciam sozinhas ao serviço nos dias de retorno. Para Marcos e Cantero (2009), a mãe é considerada como maior provedora de apoio emocional aos pacientes diagnosticados com TAs. São elas as responsáveis por acompanharem, na maior parte do tempo, as(os) filhas(os) que se encontram em situação de sofrimento, durante as consultas, exames e internações. As principais preocupações dessas mães estão relacionadas a aspectos financeiros acarretados pelo tratamento, mudanças na dinâmica familiar, dificuldades em encontrar e manter o tratamento para as(os) filhas(os), bem como os sacrifícios pessoais necessários, já que elas sentem que precisam reavaliar suas prioridades (Keitel et al., 2010; Valdanha et al, 2013a).

\section{Mudando a Percepção sobre os TAs: "Eu fui aprendendo cada vez mais o que é o transtorno alimentar"}

Com o início do tratamento especializado, as mães relatam que tomaram consciência da gravidade do quadro clínico das(os) filhas(os).

Acho, assim, que é uma coisa [transtorno alimentar], uma doença muito grave, que acaba afetando a familia inteira, a família inteira, tudo. As pessoas que vivem em torno da pessoa que tá com esse problema sofrem juntos com a pessoa, né? [...] Eu acho que é uma doença que exige muito cuidado, muito tratamento, muita dedicação. (Amanda)

Eu penso que é uma doença muito grave, teriam que dar mais atenção, né, não é uma coisa ao alcance de todos, não. Eu, graças a Deus, consegui aqui, mas é um tratamento muito caro pra quem não tem isso e não é todo mundo que enxerga que ofilho tem anorexia [...] então deve ter muita, muita gente que tem anorexia que não conseguiu perceber que ofilho tem anorexia ou que tem alguma anormalidade e pode tratar isso como uma frescura, como uma manha. (Eliana)

Tanto a fala de Amanda como a de Eliana revelam a assunção de consciência crítica em relação aos TAs, o que permite que se posicionem como cuidadoras cientes de seus direitos e conquistas, em termos de tratamento. Criticam as limitações e barreiras encontradas, como a falta de acessibilidade dos serviços e a ausência de percepção dos pais em relação aos agravos de saúde das(os) filhas(os). Amanda e Eliana valorizam o início do tratamento, quando ainda tinham uma compreensão superficial do problema e sofriam por não conseguirem deter a rebeldia das(os) filhas(os) e reverter suas restrições alimentares, evitando os comprometimentos à saúde e os diversos prejuízos clínicos. As falas dessas mães vieram acompanhadas de alívio, ao perceberem que suas filhas estavam sendo cuidadas no serviço especializado. Concomitantemente, elas se percebiam também cuidadas em suas necessidades, o que mostra a importância de encontrarem serviços e profissionais capazes de catalisarem seus anseios, com uma visão dialógica, pedagógica e terapêutica. Já Betina e Célia, cuidadoras cujas filhas tinham maior tempo de tratamento, apresentavam uma compreensão mais aprofundada das possíveis causas da $\mathrm{AN}$, por elas relacionadas aos aspectos emocionais das filhas, com reflexões que puderam ser potencializadas pelas discussões travadas no contexto dos atendimentos, na medida em que essas mães ampliam sua participação e aproveitamento dos espaços oferecidos pelos serviços.

Mas hoje a gente vê que o transtorno é decorrente de algum problema emocional, acho que não é tão distante assim, já tá bem mais próximo, mais perigoso. Não sei se é porque antes 
quase ninguém sabia, que ninguém aparentava, né, e hoje é mais conhecido. (Betina)

Hoje eu penso que é uma doença, sei lá, é uma coisa assim, sei lá, assim, acho que misturada assim com perdas, com... ou a própria família mesma, sei lá, acho que é uma maneira que eles encontram de chamar a atenção. (Célia)

A progressiva tomada de consciência acerca da problemática permite o empoderamento gradual dos familiares, que se deslocam da posição inicial de assujeitados (foram recorrentes os relatos das mães de que se sentiam "reféns" do problema das filhas) e passam a assumir a posição de experts no transtorno e copartícipes do cuidado.

Entendemos que o cuidado é indissociável da compreensão do processo saúde-doença. De acordo com Winnicott (1971/1999), a saúde e a doença são expressões da possibilidade de ser e estar no mundo. O tratamento envolve potencializar alternativas e criar as condições para que o amadurecimento possa ocorrer. Se encontrar o ambiente propício, a pessoa doente pode "encontrar uma solução pessoal para problemas complexos da vida emocional e das relações interpessoais" (Winnicott, 1984/1999, p. 114). Nessa acepção, não se trata de remover a doença, mas de cuidar da pessoa. Portanto, é preciso ir em busca de um cuidado em saúde mental que, em vez da reificação do patológico, possa conjugar os modos de vida e o desenvolvimento integral das pessoas assistidas.

Aparece também, nas falas das mães, a compreensão de que a família exerce forte influência no desenvolvimento dos TAs. Essa visão corrobora hipóteses formuladas por diversos estudos disponíveis na literatura científica nacional e internacional acerca da etiologia dessa psicopatologia (Chan \&Ma, 2004; Nilsson et al., 2009; Oliveira \& Santos, 2006; Valdanha et al., 2013b).

\section{Considerações Finais}

Neste estudo, o caminho percorrido pelas mães cujas(os) filhas(os) foram diagnosticadas com TAs apresentou-se árduo, sinuoso e pontuado por diversas barreiras e percalços. O desenho esboçado não é de um percurso linear, mas de um itinerário acidentado, marcado principalmente pelas dificuldades de diagnosticar os transtornos do comportamento alimentar devido a seus diversos determinantes, o que inclui a complexidade inerente a essa condição crônica de saúde. As dificuldades decorrem de vários fatores combinados, como a baixa percepção social e sensibilidade familiar em relação à identificação e decodificação dos sinais e sintomas sugestivos do problema, bem como a baixa qualificação técnica dos profissionais de saúde e educadores. Essas barreiras dificultam a detecção precoce e o encaminhamento dos casos suspeitos para serviços específicos. Além disso, os recursos de tratamento especializados ainda são escassos e extremamente limitados em nossa realidade, até mesmo nas capitais e outros grandes centros urbanos do país. No que diz respeito aos locais de atendimento existentes, é necessário incrementar a integração entre os serviços de saúde e redimensionar as atividades de acolhimento, tanto no nível da atenção primária, como nos níveis secundário e terciário.
Compreender o itinerário terapêutico de famílias usuárias de serviços públicos de saúde permite agregar conhecimento sobre as facilidades e barreiras encontradas no percurso empreendido pela população até que se obtenha um diagnóstico preciso e que se implemente um plano terapêutico por meio de uma equipe de saúde multidisciplinar. Acreditase que o conhecimento obtido neste estudo contribui para que possam ocorrer melhoras na oferta dos cuidados prestados ao auxiliar os serviços especializados a implementarem políticas de divulgação do tratamento junto a escolas, serviços de saúde e associações de bairro.

As mães entrevistadas descreveram diversas barreiras percebidas nos serviços da comunidade e no sistema de saúde, pois enfrentaram dificuldades em encontrar profissionais capacitados para o diagnóstico e atendimento especializado, além do tempo despendido nas filas de espera das instituições de saúde, o que parece prejudicar tanto o diagnóstico como o prognóstico dos usuários desses serviços. Observa-se, ainda, que, muitas vezes, o paciente ou familiar procura o serviço especializado após a suspeita ou diagnóstico de um outro profissional, em geral profissional de saúde ou educador. Após esse momento, acontece a busca por tratamento adequado/especializado (Favero-Nunes \& Santos, 2010).

A análise do itinerário terapêutico das famílias participantes desta pesquisa propiciou um olhar mais atento ao atendimento que vem sendo oferecido para pessoas acometidas por TAs, bem como para seus familiares. Percebe-se que é necessária maior atenção aos processos saúde/doença/cuidado, com o objetivo de compreender não apenas o percurso percorrido, mas também seus percalços e peripécias, a fim de aprimorar a qualidade do cuidado prestado e corrigir os pontos frágeis da assistência oferecida.

Também foi possível depreender, nos excertos de falas, a percepção de um número restrito de serviços especializados no tratamento de TAs, o que dificulta o acesso de pacientes e restringe as opções de tratamento, principalmente para as pessoas que residem distantes dos grandes polos urbanos ou que habitam as periferias dos grandes centros. Além da baixa disponibilidade de tais serviços especializados, devido às limitações político-institucionais que circunscrevem o setor saúde, percebe-se que existem reais dificuldades em estabelecer o diagnóstico inicial de TAs na rede pública e privada de saúde. Muitas vezes, isso acontece por despreparo dos profissionais, que não cogitam a hipótese do problema por não dominarem os conhecimentos necessários para o rastreamento; outras vezes, pode ocorrer em decorrência do comportamento dos pacientes, que, por meio da palavra e de outros artifícios, tentam camuflar os sintomas que se manifestam na alimentação e na grave distorção da imagem corporal. Para mencionar um exemplo dos estratagemas utilizados para mascarar a perda acentuada de peso durante o retorno ambulatorial, uma paciente colocava pedras e saquinhos de areia nos bolsos do casaco pouco antes de subir na balança.

De modo análogo, os relatos inverídicos no recordatório alimentar pode esconder por mais tempo os graves sintomas que se manifestam no comportamento alimentar, o que pode acarretar erros de diagnóstico e tratamento não apropriado, ou então resultar em nenhum tipo de cuidado, pois o profissional pode acreditar que não há nada de errado com o paciente sob 
avaliação. Sem saber com exatidão o que está acontecendo, a família pode continuar sua peregrinação circular pelos diferentes serviços de saúde em busca de uma resposta convincente, e o tempo despendido nessa movimentação inútil pode impactar o prognóstico, pois há melhores chances de boa evolução quando o diagnóstico acontece logo no início do quadro. Assim, é necessária uma apreciação clínica atenta aos sinais precoces ou latentes, aos indícios ditos e não ditos, além da busca pelo diálogo com os outros membros da família, que podem ser bons observadores da rotina diária da pessoa acometida.

As repercussões dos TAs e do tratamento impactam todo o sistema familiar, o que se mostrou condensado na fala de uma das participantes, que referiu que "a família inteira sofre com a pessoa que está com esse problema". Nesse contexto, vale ressaltar que os pais, muitas vezes, mostram-se frustrados e decepcionados ao se depararem com o despreparo dos profissionais consultados, que, muitas vezes, revelamse insensíveis ao sofrimento dos familiares, com pouca disponibilidade para acolherem suas angústias e contradições (Favero-Nunes, \& Santos, 2010). Desse modo, pode haver um desperdício de energia e uma despotencialização dos recursos de assistência disponíveis nos serviços de saúde.

Os aspectos apontados poderão nortear a atuação de profissionais de diversas áreas, que devem se esforçar para minimizar os possíveis entraves no diagnóstico e tratamento dos TAs. Como esses transtornos se situam em um campo de interdisciplinaridade, o conhecimento sobre o itinerário terapêutico dos pacientes pode ser de interesse para os vários profissionais que compõem a equipe de saúde. É desejável maior investimento na otimização dos recursos e na articulação mais efetiva entre os profissionais e serviços existentes na atenção básica e os ambulatórios especializados. A equipe multiprofissional responsável pelo tratamento de pacientes com AN e pelo acolhimento de seus familiares deve estar atenta à maneira como o serviço tem sido divulgado na comunidade, buscando conhecer quais são os passos percorridos anteriormente por seus usuários, bem como as dificuldades e facilidades que encontraram no périplo empreendido. $\mathrm{O}$ intuito é ampliar o alcance do tratamento para um número maior de pessoas, que, muitas vezes, são negligenciadas por falta de informações qualificadas ou impossibilidade de acesso a tratamentos específicos.

Um caminho promissor é incorporar aos serviços espaços e modalidades de cuidado terapêutico voltados para a aprendizagem participativa de mães e demais familiares, engajando-os efetivamente nos esforços de reabilitação do membro acometido, de modo a promover cidadania com o empoderamento dos cuidadores, elevando, assim, suas competências e habilidades para o manejo dos sintomas de TAs. É essencial devolver aos pais de adolescentes com TAs uma perspectiva de futuro, com a esperança de que poderão superar os desafios que obstaculizam o processo de desenvolvimento dos(as) filhos(as), de modo a fomentar expectativas favoráveis em relação ao prognóstico.

\section{Referências}

Alves, E., Vasconcelos, F. A. G., Calvo, M. C. M., \& Neves, J. (2008). Prevalência de sintomas de anorexia nervosa e insatisfação corporal em adolescentes do sexo feminino do município de Florianópolis, Santa Catarina, Brasil. Cadernos de Saúde Pública, 24(3), 503-512.

American Dietetic Association Reports. (2011). Position of the American Dietetic Association: Nutrition intervention in the treatment of eating disorders. Journal of American Dietetic Association, 111(8), 1236-1241.

American Psychiatric Association. (2006). Practice guideline for the treatment of patients with eating disorders (3thed.). Arlington, VA: American Psychiatric Publishing.

American Psychiatric Association. (2013). Diagnostic and statistical manual of eating disorders (5th ed.). Arlington, VA: American Psychiatric Publishing.

Andrade, T. F., \& Santos, M. A. (2009). A experiência corporal de um adolescente com transtorno alimentar. Revista Latinoamericana de Psicopatologia Fundamental, 12(3), 454-468.

Appolinário J. C., \& Claudino, A. M. (2000). Transtornos alimentares. Revista Brasileira de Psiquiatria, 22(2), 28-31.

Areemit, R. S., Katzman, D. K., Pinhas, L., \& Kaufman, M. E. (2010). The experience of siblings of adolescents with eating disorders. Journal of Adolescent Health, 46(6), 569-576.

Assumpção, C. L., \& Cabral, M. D. (2002). Complicações clínicas da anorexia nervosa e bulimia nervosa. Revista Brasileira de Psiquiatria, 24(3), 29-33.

Benninghoven, D., Tetsch, N., Kunzendorf, S., \& Jantscheck, G. (2007). Body image in patients with eating disorders and their mothers, and the role of family functioning. Comprehensive Psychiatry, 48(2), 118-123.

Bighetti, F., Santos, M. A., Ribeiro, R. P. P., Oliveira, E. A., Unamuno, M. R. L., \& Dos Santos, J. E. (2007). Transtornos alimentares: Anorexia e bulimia nervosas. In C. E. Kalinowski (Org.), PROENF, Programas de Atualização em Enfermagem, Saúde do Adulto. Ciclo 2, Módulo 2 (pp. 9-44). Porto Alegre: Artmed/Panamericana.

Birmingham, C. L., Su, J., Hlynsky, J. A., Goldner, E. M., \& Gao, M. (2005). The mortality rate from anorexia nervosa. International Journal of Eating Disorders, 38(2), 143-146.

Bogdan, R., \& Biklen, S. (1994). Investigação qualitativa em educação. Porto: Porto Editora.

Cabral, A. L. L. V., Martinez-Hemáez, A., Andrade, E. I. G., \& Cherchiglia, M. L. (2011). Itinerários terapêuticos: O estado da arte da produção científica no Brasil. Ciência \& Saúde Coletiva, 16(11), 4433-4442.

Cartwright, M. M. (2004). Eating disorder emergencies: Understanding the medical complexities of the hospitalized eating disordered patient. Critical Care Nursing Clinics of North America, 16(4), 515-530.

Chan, Z. C. Y., \& Ma, J. L. C. (2004). A etiology of anorexia nervosa in Hong Kong: A social work qualitative inquiry. Child and Family Social Work, 9(2), 177-186.

Claudino, A. M., \& Borges, M. B. F. (2002). Critérios diagnósticos para os transtornos alimentares: Conceitos em evolução. Revista Brasileira de Psiquiatria, 24(3), 7-12.

Cobelo, A. W., Saikali, M. O., \& Schomer, E. Z. (2004) A abordagem familiar no tratamento da anorexia e bulimia nervosa. Revista de Psiquiatria Clínica, 31(4), 184-187. 
Dallos, R., \& Denford, S. (2008). A qualitative exploration of relationship and attachment themes in families with an eating disorder. Clinical Child Psychology and Psychiatry, 13(2), 305-322.

Dos Santos, J. E. (2006). GRATA: Nossa história, trabalho e desafios. Medicina (Ribeirão Preto), 39(3), 323-326.

Dunker, K. L., \& Phillipi, S. T. (2003). Hábitos e comportamentos alimentares de adolescentes com sintomas de anorexia nervosa. Revista de Nutrição, 16(1), 51-60.

Espíndola, C. R., \& Blay, S. L. (2009). Percepção de familiares sobre a anorexia e bulimia: Revisão sistemática. Revista de Saúde Pública, 43(4), 707-716.

Favero-Nunes, M. A., \& Santos, M. A. (2010). Itinerário terapêutico percorrido por mães de crianças com transtorno autístico. Psicologia: Reflexão e Crítica, 23(2), 208-221.

Fursland, A. \& Watson, H. J. (2014). Eating disorders: A hidden phenomenon in outpatient mental health? International Journal of Eating Disorders, 47(4), 422-425. Doi: 10.1002/eat.22205.

Gazignato, E. C. S., Scorsolini-Comin, F., Souza, L. V., Kazan, A. L., \& Santos, M. A. (2008). Beleza põe mesa? Transtornos alimentares e grupo. Revista da Spagesp, 9(1), 24-32.

Gerhardt, T. E. (2006). Itinerários terapêuticos em situações de pobreza: diversidade e pluralidade. Cadernos de Saúde Pública, 22(11), 2449-2463.

Gomes, R., Moreira, M. C. N, Nascimento, E. F., Rebello, L. E. F. S., Couto, M. T., \& Schraiber, L. B. (2011). Os homens não vêm! Ausência e/ou invisibilidade masculina na atenção primária. Ciência \& Saúde Coletiva, 16(1), 983-992.

Guerin, G. D., Rossoni, E., \& Bueno, D. (2012) Itinerários terapêuticos de usuários de medicamentos de uma unidade de Estratégia de Saúde da Família. Ciência \& Saúde Coletiva, 17(11), 3003-3010.

Honey, A., Clarke, S., Halse, C, Kohn, M., \& Madden, S. (2006). The influence of siblings on the experience of anorexia nervosa of adolescent girls. European Eating Disorders Review, 14(5), 315-322.

Keitel, M. A., Parisi, M., Whitney, J. L., \& Stack, L. F. (2010). Salient stressors for mothers of children and adolescents with anorexia nervosa. Eating Disorders: The Journal of Treatment \& Prevention, 18(5), 435-444.

Latzer, Y. Ben-Ari, A., \& Galimidi, N. (2002). Anorexia nervosa and the family: Effects on younger sisters to anorexia nervosa patients. International Journal of Adolescent Medical Health, 14(4), 275-281.

Leal, G. V. S. (2013). Fatores associados ao comportamento de risco para transtornos alimentares em adolescentes na cidade de São Paulo (Unpublished doctoral dissertation), Universidade de São Paulo, São Paulo.

Leonidas, C., \& Santos, M. A. (2012). Imagem corporal e hábitos alimentares na anorexia nervosa: Uma revisão integrativa. Psicologia: Reflexão e Crítica, 25(3), 550-558.

Leonidas, C., \& Santos, M. A. (2013). Instrumentos de avaliação da imagem corporal e dos hábitos alimentares na anorexia nervosa: Análise da produção científica. Psicologia: Ciência e Profissão, 33(4), 868-883.

Leonidas, C., \& Santos, M. A. (2014) Social support networks and eating disorders: An integrative review of the literature. Neuropsychiatric Disease and Treatment, 10, 915-927.
Leonidas, C., Crepaldi, M. A., \& Santos, M. A. (2013). Bulimia nervosa: Uma articulação entre aspectos emocionais e rede de apoio social. Psicologia: Teoria e Prática, 15(2), 62-75.

Lima, N. L., Rosa, C. O. B., \& Rosa, J. F. V. (2012). Identificação de fatores de predisposição aos transtornos alimentares: Anorexia e bulimia em adolescentes de Belo Horizonte, Minas Gerais. Estudos e Pesquisas em Psicologia, 12(2), 360-378.

Mattosinho, M. M. S., \& Silva, D. M. G., V. da. (2007). Itinerário terapêutico do adolescente com diabetes mellitus tipo 1 e seus familiares. Revista Latinoamericana de Enfermagem, 15(6), 1113-1119.

Marcos, Y. Q., \& Cantero, M. C. T. (2009). Assesment of social support dimensions in patients with eating disorders. The Spanish Journal of Psychology, 12(1), 226-235.

Marques, A. L. M., \& Mângia, E.F. (2013).Therapeutic itineraries of individuals with problems consequent to harmful use of alcohol. Interface: Comunicação, Saúde, Educação, 17(45), 433-444.

Morgan, C. M., \& Claudino, A. M. (2005). Epidemiologia e etiologia. In A. M. Claudino, \& M. T. Zanella (Orgs.), Transtornos alimentares e obesidade (pp. 15-23). Barueri, SP: Manole.

Morgan, C. M., Vecchiatti, I. R., \& Negrão, A. B. (2002) Etiologia dos transtornos alimentares: Aspectos biológicos, psicológicos e sócio-culturais. Revista Brasileira de Psiquiatria, 24(3), 18-23.

Nilsson, K., Abrahamsson, K., Torbiornsson, A., \& Hägglöff, B. (2009). Causes of adolescent onset anorexia nervosa: patient perspectives. Eating Disorders, 15(2), 125-133.

Nunes, M. A. (2006). Epidemiologia dos transtornos alimentares. In M. A. Nunes, J. C. Appolinario, A. L. Galvão, \& W. Coutinho (Orgs.), Transtornos alimentares e obesidade (pp. 51-58). Porto Alegre: Artmed.

Oliveira, E. A., \& Santos, M. A. (2006). Perfil psicológico de pacientes com anorexia e bulimia nervosas: A ótica do psicodiagnóstico. Medicina (Ribeirão Preto), 39(3), 353-360.

Organização Mundial de Saúde (1993). Classificação de transtornos mentais e de comportamento da CID-10. Descrições clínicas e diretrizes diagnósticas. Porto Alegre: Artes Médicas.

Palma, R. F. M., Dos Santos, J. E., \& Ribeiro, R. P. P. (2013). Hospitalização integral para tratamento dos transtornos alimentares: A experiência de um serviço especializado. Jornal Brasileiro de Psiquiatria, 62(1), 31-37.

Pinzon, V., \& Nogueira, F. C. (2004) Epidemiologia, curso e evolução dos transtornos alimentares. Revista de Psiquiatria Clínica, 31(4), 676-682.

Polivy, J., \& Herman, C. (2002). Causes of eating disorders. Annual Review of Psychology, 53, 187-213.

Quiles-Cestari, L. M., \& Ribeiro, R. P. P. (2012).The occupational roles of women with anorexia nervosa. Revista LatinoAmericana de Enfermagem, 20(2), 235-242.

Rosa, B. P., \& Santos, M. A. (2011). Comorbidade entre bulimia e transtorno de personalidade borderline: Implicações para o tratamento. Revista Latinoamericana de Psicopatologia Fundamental, 14(2), 268-282.

Saikali, C. J., Soubhia, C. S., Scalfaro, B. M., \& Cordas, T. A. (2004). Imagem corporal nos transtornos alimentares. Revista de Psiquiatria Clínica, 31(4), 164-166. 
Santana, M. L. P., Ribeiro Junior, H. C., Giral, M. M., \& Raich, R. M. (2012). La epidemiología y los factores de riesgo de los trastornos alimentarios en la adolescência: Una revisión. Nutrition Hospital, 27(2), 391-401.

Scorsolini-Comin, F., Souza, L. V., \& Santos, M. A. (2010). A construção de si em um grupo de apoio para pessoas com transtornos alimentares. Estudos de Psicologia (Campinas), 27(4), 467-478.

Souza, L. V., \& Santos, M. A. (2007). Anorexia e bulimia: Conversando com as famílias. São Paulo: Vetor.

Souza, L. V., \& Santos, M. A. (2009). A construção social de um grupo multifamiliar no tratamento dos transtornos alimentares. Psicologia: Reflexão e Crítica, 22(3), 317-26.

Souza, L. V., Santos, M. A., \& Scorsolini-Comin, F. (2009). Percepções da família sobre a anorexia e bulimia nervosa. Vinculo, 1(6), 26-38.

Souza, L. V., \& Santos, M. A. (2010). A participação da família no tratamento dos transtornos alimentares. Psicologia em Estudo (Maringá), 15(2), 285-294.

Souza, L. V., \& Santos, M. A. (2014). Transtorno alimentar e construção de si no relacionamento profissional-usuário. Psicologia e Sociedade, 26(2), 506-516.
Spink, M. J. P., Medrado, B., \& Mello, R. P. (2002). Perigo, probabilidade e oportunidade: A linguagem dos riscos na mídia. Psicologia: Reflexão e Crítica, 15(1), 151-164.

Stake, R. E. (2000). Case studies. In N. K. Denzin, \& Y. S. Lincoln (Orgs.), Handbook of qualitative research (pp. 435-454). London: Sage.

Turato, E. R. (2005). Métodos qualitativos e quantitativos na área da saúde: Definições, diferenças e seus objetos de pesquisa. Revista de Saúde Pública, 39(3), 507-514.

Valdanha, E. D., Scorsolini-Comin, F., \& Santos, M. A. (2013a). Anorexia nervosa e transmissão psíquica transgeracional. Revista Latino-Americana de Psicopatologia Fundamental, 16(1), 71-88.

Valdanha, E. D., Scorsolini-Comin, F., Peres, R. S., \& Santos, M. A. (2013b). Influência da família na anorexia nervosa: em busca das melhores evidências científicas. Jornal Brasileiro de Psiquiatria, 62(3), 225-233.

Winnicott, D. W. (1971). O conceito de indivíduo saudável. In Tudo começa em casa (pp. 3-22; J. L. Camargo, Trad.). São Paulo: Martins Fontes. (Original publicado em 1999)

Winnicott, D. W. (1984). Variedades de psicoterapia. In Privação e delinquência (pp. 237-245; A. Cabral, Trad.). São Paulo: Martins Fontes. (Original publicado em 1961)

Recebido em 23.03.2014

Primeira decisão editorial em 29.04.2015

Versão final em 12.05.2015

Aceito em 04.08.2015 